\title{
Proline-catalysed asymmetric ketol cyclizations: The template mechanism revisited
}

\author{
R MALATHI, ${ }^{\mathrm{a}}$ D RAJAGOPAL, ${ }^{\mathrm{b}}$ ZOLTAN G HAJOS $^{\mathrm{c}}$ and $\mathrm{S}$ SWAMINATHAN* ${ }^{\mathrm{b}}$ \\ ${ }^{a}$ Department of Crystallography and Biophysics, University of Madras, Chennai 600 025, India \\ ${ }^{b}$ Department of Organic Chemistry, University of Madras, Chennai 600 025, India \\ ${ }^{c}$ Hoffmann-La Roche Inc, Nutley, NJ 07110, USA; Present address: 802A, Pompton Road, \\ Monroe Township, NJ 08831-4234, USA \\ e-mail: swaminathan1923@yahoo.co.in
}

MS received 8 December 2003; revised 16 April 2004

\begin{abstract}
A modified template mechanism based on modelling studies of energy minimised complexes is presented for the asymmetric proline-catalysed cyclization of triketones $\mathbf{1}, \mathbf{2}$ and $\mathbf{3}$ to the $2 S, 3 S$-ketols 1a, 2a and 3a respectively. The template model involves a three-point contact as favoured in enzymesubstrate interactions. Our minimisation studies are in agreement with the divergent behaviour of the 6,5-, 6,6- and 6,7-bicyclic systems. They support the high 93.4\% ee observed with the 6,5-bicyclic ketol and the lower $73 \%$ ee found with the 6,6-bicyclic ketol. The calculations also explain the lack of asymmetric induction with the 6,7-bicyclic system.
\end{abstract}

Keywords. Proline-catalysed ketol cyclization; template mechanism.

\section{Introduction}

A recent publication ${ }^{1}$ has highlighted the increasing use of proline by calling it "the simplest enzyme" (it was named so earlier by one of us in $1974^{2}$ ) to effect asymmetric transformations with high enantio selectivities. The earliest use of proline in asymmetric synthesis was in the synthesis of the optically active Hajos-Parrish ketol (1a) $)^{2,3}$ and the WielandMiescher ketone (2b) ${ }^{3,4}$ In a series of papers, List $e t$ $a l^{5-7}$ and also MacMillan and Northrup ${ }^{8}$ have reportted enantioselective aldol addition reactions of acetone and substituted acetones with aldehydes catalysed by proline. Conflicting kinetic evidence has been obtained by Agami et $a l^{9}$ for the participation of two molecules of proline in the transition states of proline-catalysed intramolecular cyclizations and by Houk et $a l^{10}$ for the involvement of only one molecule of proline in such reactions. Both groups of workers have preferred the enamine mechanism to the carbinolamine mechanism (original $^{2}$ and modified ${ }^{11}$ ) and also the template mechanism. ${ }^{12}$ We present here a modified version of the template mechanism based on modelling studies of energy minimised complex for the cyclization of the

\footnotetext{
*For correspondence
}

three triketones $\mathbf{1}, \mathbf{2}$ and $\mathbf{3}$ to the ketols 1a, 2a and 3a respectively.

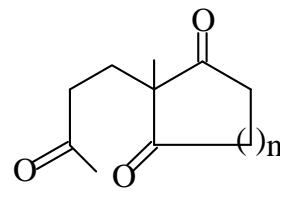

1, $n=1$

2, $n=2$

3, $n=3$

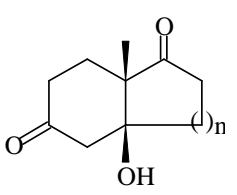

1a, $n=1$

2a, $n=2$

3a, $n=3$

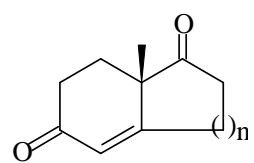

1b, $n=1$

2b, $n=2$

3b, $n=3$
On the basis of evidence adduced already, ${ }^{12}$ we assume that the asymmetric cyclizations take place on the crystal lattice of $S$-proline. This was taken from the literature; ${ }^{13}$ the structures of substrates $(\mathbf{1}$, 2 and 3) were constrained with one or two proline moieties in the lattice to obtain specific hydrogen bonds while keeping the coordinates of proline fixed. The triketone models (built using Builder software in INSIGHT II) are of random choice, since while complexing with proline, these random models may be expected to assume an energy minimised state to fulfill the hydrogen bonding constraints. The models were minimised in energy using force field CFF91 using DISCOVER software for the formation of both $2 S, 3 S$-ketol and $2 R, 3 R$ ketol for each of the triketones $\mathbf{1}, \mathbf{2}$ and $\mathbf{3}$. Com- 

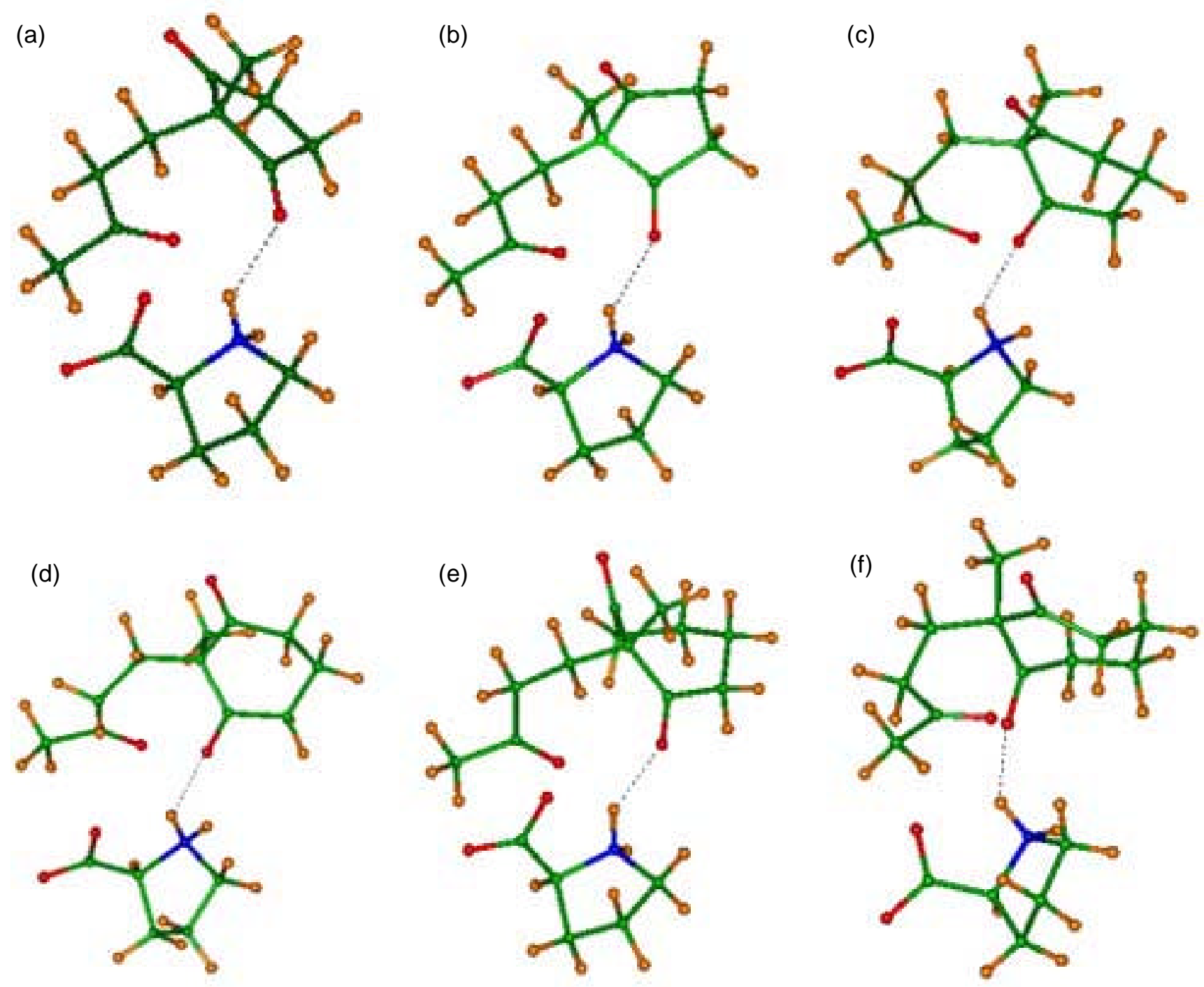

Figure 1. Complexes involving one-proline molecule and the substrates (1, 2 and 3) respectively, for formations of $S$ (a, $\mathbf{c}$ and $\mathbf{e})$ and $R(\mathbf{b}, \mathbf{d}$ and $\mathbf{f})$ ketols.

plexes involving one proline molecule each are shown in figure $1 \mathrm{a}-\mathrm{f}$ respectively and complexes involving two proline molecules each are shown in figure $2 \mathrm{a}-\mathrm{f}$ respectively.

\section{Results and discussion}

The minimum energies and their difference in the formation of complexes involving one proline and two prolines for formation of $S$ and $R$ ketols are given in tables 1 and 2 respectively.

The minimum energy values in both tables are roughly in accord with experimental findings, viz. $100 \%$ chemical yield and $93.4 \%$ optical yield of
$2 S, 3 S$-ketol $\mathbf{1} \mathbf{a}^{2}$ in the cyclization of $\mathbf{1} ; 80 \%$ chemical yield and $73 \%$ optical yield of $2 S, 3 S$-ketol $\mathbf{2} \mathbf{a}^{3}$ in the case of $\mathbf{2}$ and only $d l$-ketone $\mathbf{3 b}$ (20-25\% yield) in the case ${ }^{14}$ of $\mathbf{3}$. However, complexes as shown in figure 2 involving two prolines seem preferable since the second proline helps in the deprotonation of the side-chain methyl by forming a hydrogen bond with the adjacent carbonyl, unlike in complexes shown in figure 1 where deprotonation of an unactivated $-\mathrm{COCH}_{3}$ by the weakly basic $-\mathrm{COO}^{-}$is implied. Furthermore, the two proline complexes involve a three-point contact ${ }^{15}$ favoured in enzyme-substrate interactions. The reactions subsequent to the complexation in the 2-proline complexes may involve 
(a)
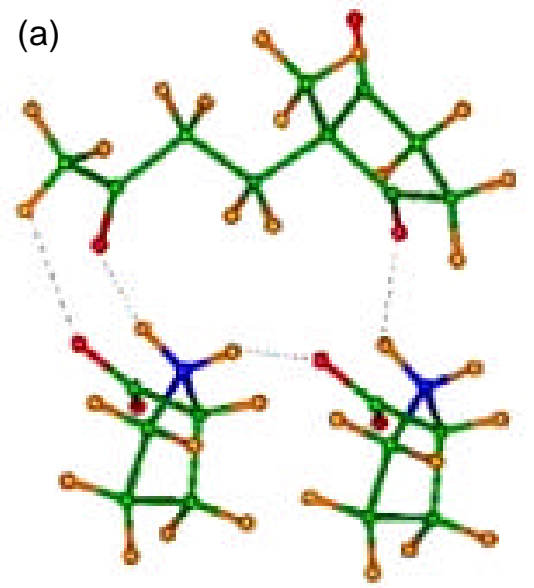

(d)

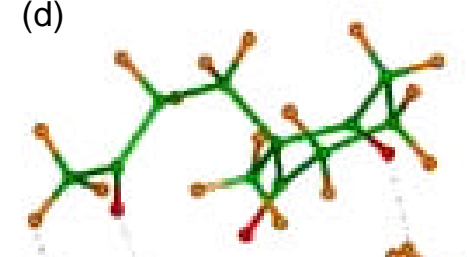

(b)

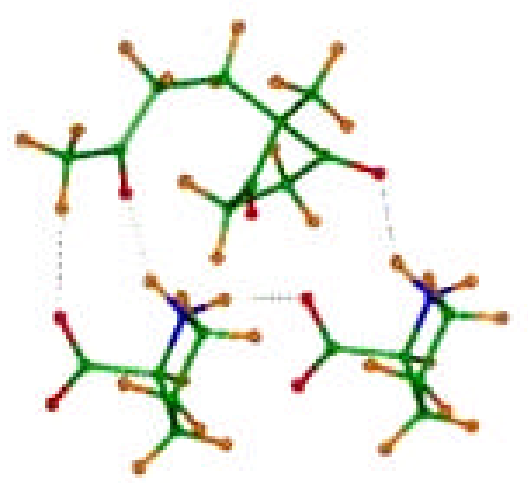

(e)
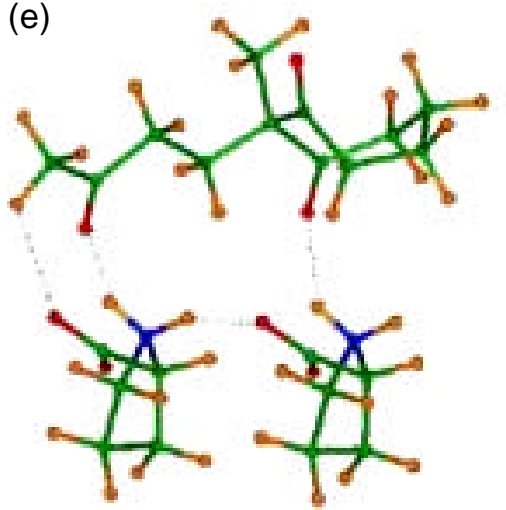

(c)

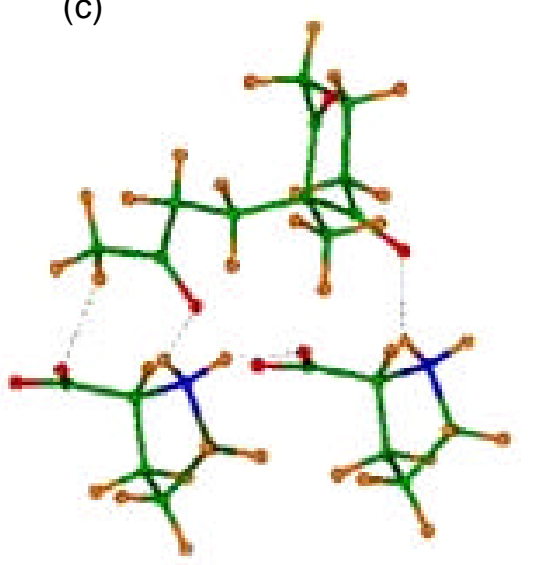

(f)

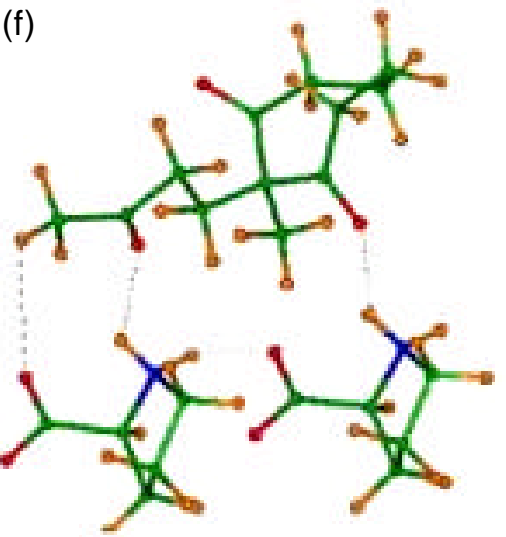

Figures 2. Complexes involving two-proline molecules and the substrates $(\mathbf{1}, \mathbf{2}$ and $\mathbf{3})$ respectively for formations of $S(\mathbf{a}, \mathbf{c}$ and $\mathbf{e})$ and $R(\mathbf{b}, \mathbf{d}$ and $\mathbf{f})$ ketols.

Table 1. One-proline complexes.

\begin{tabular}{lcccc}
\hline Complex in figure 1 & Ketol & Configuration & Minimum energy (kcals) & Difference in energy (kcals) \\
\hline a & 1a & $2 S, 3 S$ & -6.33 & -6.26 \\
$\mathrm{~b}$ & $\mathbf{1 a}$ & $2 R, 3 R$ & -0.07 & -3.88 \\
$\mathrm{c}$ & $\mathbf{2 a}$ & $2 S, 3 S$ & -4.93 & \\
$\mathrm{~d}$ & $\mathbf{2 a}$ & $2 R, 3 R$ & -1.05 & -2.21 \\
$\mathrm{e}$ & $\mathbf{3 a}$ & $2 S, 3 S$ & -6.64 & \\
$\mathrm{f}$ & 3a & $2 R, 3 R$ & -4.43 & \\
\hline
\end{tabular}

Table 2. Two-proline complexes.

\begin{tabular}{lcccc}
\hline Complex in figure 2 & Ketol & Configuration & Minimum energy (kcals) & Difference in energy (kcals) \\
\hline a & $\mathbf{1 a}$ & $2 S, 3 S$ & $-7 \cdot 51$ & $-35 \cdot 78$ \\
$\mathrm{~b}$ & $\mathbf{1 a}$ & $2 R, 3 R$ & 28.27 & \\
$\mathrm{c}$ & $\mathbf{2 a}$ & $2 S, 3 S$ & $-11 \cdot 8$ & $-20 \cdot 84$ \\
$\mathrm{~d}$ & $\mathbf{2 a}$ & $2 R, 3 R$ & $9 \cdot 04$ & -0.84 \\
$\mathrm{e}$ & $\mathbf{3 a}$ & $2 S, 3 S$ & $-10 \cdot 50$ & \\
$\mathrm{f}$ & $\mathbf{3 a}$ & $2 R, 3 R$ & $-9 \cdot 66$ & \\
\hline
\end{tabular}


a concerted cyclization to the ketols, or more likely a two-step process involving side-chain enol formation followed by $\mathrm{C}-\mathrm{C}$ bond formation. One of these steps must be rate-determining to be consistent with one-mole proline kinetics. ${ }^{10}$

\section{Acknowledgements}

We thank Professors N Yathindra and S S Rajan for their help. RM thanks the Council of Scientific and Industrial Research, New Delhi for an associateship.

\section{References}

1. Movassaghi M and Jacobsen E N 2002 Science 298 1904

2. Hajos Z G and Parrish D R 1974 J. Org. Chem. 39 1615

3. Hajos Z G and Parrish D R 1971 German Patent DE 2102623
4. Eder U, Sauer G and Wiechert R 1971 Angew. Chem., Int. Ed. (Engl.) $\mathbf{1 0} 496$

5. List B, Lerner R A and Barbas C F III $2000 \mathrm{~J}$. Am. Chem. Soc. 1222395

6. List B 2002 J. Am. Chem. Soc. 124 5656, and earlier papers cited therein

7. List B 2002 Tetrahedron 585573

8. Northrup A B and MacMillan D W C $2002 \mathrm{~J}$. Am. Chem. Soc. 1246798

9. Agami C, Puchot C and Sevestre H 1986 Tetrahedron Lett. 271501

10. Hoang L, Bahmanyar S, Houk K N and List B 2003 J. Am. Chem. Soc. 12516

11. Hajos Z 2002 http://preprint.chemweb.com/orgchem/ 0209001, Sept 5

12. Rajagopal D, Moni M S, Subramanian S and Swaminathan S 1999 Tetrahedron: Asymmetry 101631

13. Shamala N, Row T N G and Venkatesan K 1976 Acta Crystallogr. B32 3267

14. Rajagopal D $1999 \mathrm{Ph}$ D Studies in asymmetric syntheses, thesis, University of Madras, Chennai

15. Florkin M and Statz G (eds) 1964 In Comprehensive biochemistry 12237 